\title{
Multiobjective Differential Evolution with Discrete Elite Guide in Internet of Vehicles Roadside Unit Deployment
}

\author{
Feng Wang $\mathbb{D}^{1,2}$ Chenle Wang $\mathbb{D}^{3},{ }^{3}$ Kan Wang $\mathbb{D},{ }^{1}$ Qiaoyong Jiang $\mathbb{D}^{1},{ }^{1}$ Bin Wang $\mathbb{D},{ }^{1}$ \\ and Wenjuan $\mathrm{He}$ (i) $^{1}$ \\ ${ }^{1}$ School of Computer Science and Engineering, Xi'an University of Technology, Xi'an 710048, China \\ ${ }^{2}$ Shaanxi Key Laboratory of Network Computing and Security Technology, Xi'an University of Technology, Xi'an 710048, China \\ ${ }^{3}$ International Engineering College, Xi'an University of Technology, Xi'an 710048, China
}

Correspondence should be addressed to Kan Wang; wangkan@xaut.edu.cn

Received 1 October 2021; Accepted 28 October 2021; Published 11 November 2021

Academic Editor: Renchao Xie

Copyright (c) 2021 Feng Wang et al. This is an open access article distributed under the Creative Commons Attribution License, which permits unrestricted use, distribution, and reproduction in any medium, provided the original work is properly cited.

In a vehicular ad hoc network (VANET), roadside units (RSUs) are installed at roadside and intersections to process vehicle-to-infrastructure communication, collect and analyse intelligent vehicle traffic data, send information to vehicles, and achieve early warning of safe driving of vehicles. Owning to the high cost of implementing and maintaining RSUs, it is of vital importance to determine where and how many RSUs to deploy. Optimal RSU deployment requires both a small number of RSUs and the maximum coverage of vehicle running process, which constitutes a conflicting multiobjective problem. Nevertheless, existing works do not explicitly utilize multiobjective algorithm to solve the RSU deployment problem. Therefore, a multiobjective differential evolution approach is proposed in this work to solve the problem. Firstly, to conquer the complexity of urban road RSU deployment, the static model is established. Secondly, in the proposed multiobjective differential evolution with discrete elitist guide (MODE-deg), the sigmoid function is applied to discrete individual values. Finally, elitist individuals are selected based on crowding distance ranking and nondominated ranking to generate new individuals, which further improve the convergence speed and population performance. Experimental results show that MODE-deg can generate the optimal nondominant solution set with good convergence and diversity, in contrast to other multiobjective evolutionary algorithms in five test functions of ZDT.

\section{Introduction}

With the progress of science and technology, the exponential growth of vehicles has led to traffic jams and accidents. In 2003 ITU telecommunication standardization sector ITU-T automobile communication standardization conference, experts from various countries proposed that vehicular ad hoc network (VANET) [1] is expected to reduce the loss caused by traffic accidents by $50 \%$. VANET is widely used in vehicle navigation system, route planning, and other aspects by continuously collecting road vehicle operation conditions. For example, through the analysis of the volume of traffic flow, VANET can generate the optimal route for the vehicle, such that the vehicle can reach the destination in the shortest time. At the same time, VANET data is not only used for the reasonable setting of traffic light time but also used for the statistics of the number of vehicles on the road every day. In the VANET, on-board unit (OBU) [2] installed on the vehicle and road-side unit (RSU) [3] deployed on the roadside are two core components. Both OBU and RSU have wireless communication modules that support the wireless information transmission of vehicles when moving. Through the wireless communication module, the vehicle and the road test unit can exchange information. RSU could process vehicle requests, allow vehicle access to the network, and forward vehicle information. The modes of VANET are mainly divided into two types: vehicle-to-vehicle (V2V) communication [4] and vehicle-to-RSU (V2R). 
$\mathrm{V} 2 \mathrm{~V}$ communication refers to real-time transmission of observed data between vehicles in the process of vehicle driving. More explicitly, vehicles communicate directly through OBU, and vehicles must be within the communication range of both sides. However, as the vehicle drives out of the range under certain circumstances, the V2V communication link will be disconnected, which may result in data transmission delay or even transmission failure. On the other hand, V2R is the real-time communication $[5,6]$ between the OBU installed in the vehicle and the RSU deployed on the roadside. When the vehicle enters the communication range of the RSU, the RSU communicates with the vehicle in real time and then sends the obtained vehicle information to the Internet platform. The platform analyses the data and then sends it to passing vehicles via RSU. Therefore, the RSU plays a very important role in improving traffic patency and safety. The RSU allows data communication with the vehicle within its communication range and forwards vehicle information to other vehicles within its range. Thus, the deployment of RSUs could be categorized as:

(1) Deploy RSU at the intersection with large traffic flow

The number of vehicles varies greatly by region. In highly developed cities, the downtown is prosperous, and the traffic flow is relatively large. The RSU is deployed at the intersection which must be passed by vehicles, and passing vehicles have to communicate with the RSU. Therefore, deploying RSU at intersections with heavy traffic can improve the utilization rate of RSU.

\section{(2) Deploy RSU in different sections}

Deploying RSU in slow traffic areas such as the community and schools can the improve RSU utilization. However, when the RSU is deployed on highways, the vehicle speed is fast, and the vehicle topology changes rapidly. Therefore, the deployment of RSU should be tailored on the situation of different scenarios.

Some excellent works have emerged to study the RSU deployment with diverse objectives. In particular, Wang et al. [7] utilized the linear programming to solve the RSU deployment in centrality, with the goal to maximize the total centrality of RSU deployment location candidates under a given budget constraint. In order to achieve the maximum effective traffic coverage ratio under given conditions, Cai et al. [8] proposed a two-layer improved greedy algorithm to deploy roadside cable connection units and UAV auxiliary positioning system. More explicitly, Kim et al. [9] abstracted urban area maps into grid maps and proposed three different RSU deployment strategies: static, mobile but uncontrollable, and fully controllable, and polynomial running time approximation algorithm was used. Shareeda et al. [10] proposed a genetic algorithm based on RSU positioning to find the optimal or near-optimal solution. Lamb and Agrawal [11] proposed a technique for selecting context-important locations, which checks intersection vehicle statistics in the area passed by commuters most frequently and selects the most commonly used intersection location. Jian and Yang [12] proposed a significance ranking model for RSU positioning and three calculation strategies for significance degree and studied the influence strategies of RSU quantity and OBE market penetration on traffic monitoring evaluation measures. $\mathrm{Ni}$ et al. [13] constructed a maximization model of total revenue based on RSU deployment by designing a clustering algorithm based on linear programming to realize RSU deployment. Chi et al. [14] proposed a RSU layout method based on cross priority and applied three different algorithms, greedy, dynamic, and hybrid, to realize the optimal deployment of RSU in cities. Cao et al. [15] proposed an optimized deployment scheme based on the large-scale vehicle trajectory data, where $K$-nearest neighbours and branch-andbound algorithms were proposed to obtain the optimal deployment of RSU. In a word, the above research work mainly applies the algorithm to realize the deployment of RSUs.

Other excellent works have focused on RSU communication and location. Cao et al. [16] analysed the onedimensional RD problem, combining greedy thought with dynamic programming. On the other hand, in line with the requirements of minimum deployment cost and low delay of RSU, Trullols et al. [17] proposed the maximum coverage method for information transmission in intelligent transportation system, in which the dissemination points and heuristic algorithm were used to realize the communication of vehicles. Lochert et al. [18] proposed a road network infrastructure support unit layout optimization method based on genetic algorithm, by estimating travel time savings from active communication infrastructure location vectors via simulation that separates mobile and network problems from application behaviour. Ou et al. [19] proposed a connection-oriented maximum coverage RSU deployment approach, which improves the communication performance of urban areas by deploying fewer RSU. Eftekhari et al. [20] proposed a new binary planning approach, in which RSU are deployed alongside roads to maximize the transmission of information to vehicles. Anbalagan et al. [21] proposed the memetic-based RSU placement algorithm to reduce communication delay and increase the coverage area between devices in IoV. Sankaranarayanan et al. [22] proposed an optimized travel time model based on RSU to estimate the travel time of the section and used genetic algorithm to calculate the optimal number of RSU and place them in the correct position. In summary, optimizing the transmission of information [23] between vehicles and RSUs is the main work of the authors above.

The aforementioned works imply that, due to the high cost of deployment and maintenance as well as the limited communication radius, RSUs are not conducive to largescale implementation. Therefore, the deployment location and number of RSUs are extremely important. Existing work regarding RSU deployment schemes can be roughly divided into three categories: mathematical models, heuristic algorithms with game theory, and graph theory. Although greedy algorithm, genetic algorithm, and other ones are used in aforementioned works [7-22] to solve the RSU deployment problem, multiobjective optimization algorithm is not leveraged to solve multiple conflicting ones. The deployment of RSU often involves multiple conflicting objectives, thus facilitating the utilization of multiobjective evolutionary 
algorithm to solve this issue. Thus, the main contributions of the paper are as follows.

(1) RSU Deployment Model. To conquer the complexity of RSU deployment on urban roads, this paper establishes a static model, which can achieve the maximum coverage of road crossings, while ensuring the minimum number of RSU

(2) Population Dispersion. For the RSU deployment problem, the sigmoid function is applied to deal with individual values in the population. The individual dimension value is set to 1 when the RSU is deployed at the intersection and 0 otherwise

(3) Elitist Guide. Before the crossover operation of MODE-deg algorithm, firstly, the population is sorted in descending order according to the crowding distance of the population; secondly, the population is sorted in nondominant order according to the Pareto level of the population; finally, the sorted elitist individuals are selected as the base vector and terminal vector. Since the elitist retains the genes of the excellent individuals, the population convergence rate can be accelerated

In the remainder of the paper, we briefly introduce several of differential evolution algorithm and multiobjective evolution algorithm in Section 2. Thereafter, in Section 3, we describe the proposed the RSU deployment model and the MODE-deg algorithm in details. Section 4 presents simulation results of MODE-deg and compares them with three other elitist MOEAs (NSGA-II, MOEA/D, and MOEA/Darg). Meanwhile, MODE-deg is applied to solve the RSU deployment problem in urban highway scenarios. Finally, we outline the conclusions.

\section{Preliminary}

In real life, we often encounter some optimal decision problems, which can be modelled as a global optimization problem. That is, in a given feasible region and a predefined objective function on the set, the maximum or minimum value of the function in the range of the set is solved.

A multiobjective optimization problem (MOP) is a mathematical problem involving simultaneous optimization of multiple objective functions. The various subobjectives of MOP [24] are contradictory. The improvement of one subobjective may cause the performance degradation of another or several subobjectives; that is, it is impossible to achieve the optimal value of multiple subobjectives at the same time, and only coordination and compromise can be carried out among them, such that each subobjective can achieve the optimization as far as possible. The essential difference between multiobjective and single-objective optimization problem is that its solution is not unique, with a set of optimal solutions composed of many Pareto optimal solutions. Thus, each element in the set is called Pareto optimal solution or noninferior optimal solution.
The basic idea of multiobjective optimization algorithm is to optimize the function through the evolution of population. The mathematical properties of function are continuity, differentiability, convexity, and other properties, and only the value of the objective function can be calculated. Therefore, the multiobjective optimization algorithm can solve the multiobjective problem well.

Differential evolution (DE) algorithm [25] was proposed by Rainer Storm and Kenneth Price in 1997 on the basis of genetic algorithm (GA) and other evolutionary ideas, which a classical multiobjective evolutionary algorithm (MOEA) that aims at finding a finite number of solutions to approximate the real solution set. The core operation of DE mainly includes three parts: mutation, crossover, and selection.

In particular, mutation is a linear operation of multiple individuals selected from NP populations to create a mutant vector individual, and there are many mutation mechanisms of DE. In equation (1) and equation (2), $X$ is the vector population, $V$ is the mutant vector population, $i$ is the $i$ th vector individual, $G$ is the $g$ generation population, and best is the optimal individual in the $G$ generation population, respectively. The randomly chosen indexes from $r 1, r 2, r 3 \in\{1,2 \cdots N P\}$ are mutually different integers, and $F$ is the mutation operator.

\subsection{Mutation.}

$$
\begin{aligned}
& \mathrm{DE} / \mathrm{rand} / 1: V_{i}(g)=X_{r 1}(g)+F \cdot\left(X_{r 2}(g)-X_{r 3}(g)\right) \\
& \text { DE/best/1 }: V_{i}(g)=X_{\text {best }}(g)+F \cdot\left(X_{r 2}(g)-X_{r 3}(g)\right) .
\end{aligned}
$$

\subsection{Crossover.}

$U_{j i}(g+1)=\left\{\begin{array}{l}V_{j i}(g+1) \text { if } \operatorname{rand}(0,1)<\operatorname{Cr} \text { or } \mathrm{j}=\operatorname{Randi}(0, \mathrm{D}) \\ X_{j i}(g+1) \text { else. }\end{array}\right.$

In equation (3), rand $(0,1)$ is a random number from 0 to $1, \mathrm{Cr}$ is a crossover constant, $D$ is the individual vector dimension, $j$ is a certain dimension of the individual vector, and Randi $\in(1, D)$ are a random integer between 0 and $D$, respectively.

\subsection{Selection.}

$$
X_{i}(g+1)= \begin{cases}V_{i}(g) & \text { if } f(g)<f\left(X_{i}(g)\right) \\ X_{i}(g) & \text { else }\end{cases}
$$

To decide a member of generation $g+1$, the trial vector yields compared to the target vector using the greedy criterion. If the trial vector yields a smaller cost function value than the target vector, then the trial vector is selected as the next generation; otherwise, the old value $x$ is retained. After many generations of evolution, the optimal solution is finally obtained. 
A MOP is mathematically described as:

$$
\min F(x)=\left[f_{1}(x), f_{2}(x), \cdots, f_{k}(x)\right]
$$

In equation (5), $x=\left(x_{1}, x_{2}, x_{3} \cdots x_{d}\right) \in \Omega$. The dimension of candidate solutions is $d$.

More especially, the decision space is $\Omega=\left\{x \in R \mid h_{j}(x)\right.$ $\leq 0, j=1,2, \cdots, k\}, R_{k}$ is called the objective space, $F: \Omega$ $\longrightarrow R_{k}$ consists of $k$ real-valued objective functions, and $h(x)$ is a continuous function, respectively.

For Pareto dominance relation, if the optimal solution optimization problem is solved with the minimum target value of the vector, and $x_{b}$ is greater than or equal to the objective function value of $x_{a}$ for all objective functions, then $x_{a}$ dominates $x_{b}\left(x_{a} \prec x_{b}\right)$.

If there is a group variable $x^{*}$ that is not dominated by any variable, $x^{*}$ is said to be a nondominated solution. The solutions in Pareto optimal solution set (PS) do not dominate each other; then, such a set of solutions is called Pareto optimal solution set of multiobjective problems, PS $=\{x * \epsilon$ $\left.\Omega \mid \nexists x \in \Omega, F(x)<F\left(x^{*}\right)\right\}$. The set corresponding to PS in the target space is called Pareto-optimal front $(P F)$, $P F=\{F(x) \mid x \in P S\}$.

In different evolutionary mechanisms, MOEA can be divided into three categories: dominated MOEA [25-29], which selects all nondominant individuals by nondominant ranking and maintains population diversity by crowding distance ranking; decomposition-based MOEA [30-32], which decomposes the MOP into $n$ scalar optimization subproblem and solves them in a cooperative way; and indicatorbased MOEA [33, 34], which utilizes evaluation indicators to guide the search process and solution selection process, to achieve better convergence and diversity.

\section{MODE-deg}

This paper studies the deployment of RSU in urban road intersections to achieve optimal deployment of RSU. RSUs are typically deployed at traffic light intersections. By deploying as few RSUs as possible, we can achieve as much intersection coverage as possible, and RSU covers not only intersections but also the corresponding sections, which improves the ability of vehicles to obtain traffic information in a timely manner and ensures vehicle safety and smooth roads.

In Figure 1, the RSU deployment is mainly used at each intersection, since the RSU deployment at the intersection can maximize its communication radius $R$ and improve the utilization rate of RSU. As the premise of discussing the problem is the joint communication mode, only some intersections deploy RSUs, while others on the opposite. When a vehicle passes an intersection where RSU is deployed, it can communicate with RSU on vehicle-road; otherwise, it can communicate with RSU on vehicle-road.

3.1. Deployment Plan. We take the urban area within the city wall of Xi'an as the research object and establish a static model. There are $n$ intersections set $C=\left(c_{1}, c_{2}, c_{3} \cdots c_{n}\right)$ in urban area, and $D_{n * n}$ is the adjacency matrix vector.

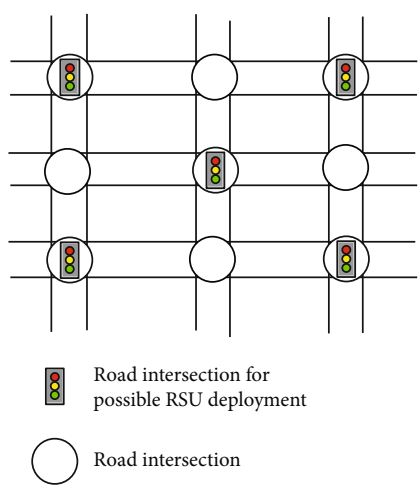

FIGURE 1: RSU deployment scenario.

Furthermore, the elements $d_{i, j}(1 \leq i \leq n, 1 \leq j \leq n)$ are the distance from the intersection $i$ to the intersection $j$, and $X$ is the set of intersections where the RSU is currently placed. The mathematical model is as follows.

$$
\begin{aligned}
& \min f_{1}=\sum_{i=1}^{n} x_{i}, \\
& \min f_{2}=1-\frac{\left|\mathrm{C}_{s}\right|}{m}, \\
& \text { s.t. } x_{i} \in\{0,1\}, \forall s, i \in\{1,2,3 \cdots \mathrm{n}\}, 0 \leq \sum_{i=1}^{n} x_{i} \leq n,
\end{aligned}
$$

where

$D_{n * n}=\left[\begin{array}{ccc}d_{1,1} & \cdots & d_{1, n} \\ d_{2,1} & \cdots & d_{2, n} \\ \cdots & \ddots & \cdots \\ d_{n, 1} & \cdots & d_{n, n}\end{array}\right], d_{i, j}= \begin{cases}0 & i=j, \\ -1 & i \text { and } j \text { are not on the same street, } \\ w & i \text { and } j \text { are on the same street. }\end{cases}$

In equation (6), a total of FL RSU side units are deployed in this scenario, and whether to deploy RSU is determined according to the value of sigmoid $S\left(x_{i}\right)$. If $S\left(x_{i}\right)$ is greater than or equal to 0.5 , then RSU is deployed at $C_{i}$; otherwise, $C_{i}$ does not deploy RSUs. In equation (6), $C_{s}$ is the number of all intersections covered by RSU communication radius, $M$ is the total number of intersections, and $f_{2}$ is the percentage of streets that are not covered, respectively. Equation (7) is the adjacency matrix of the intersection. Given $i=j$, the distance is 0 . When intersection $i$ and intersection $j$ are not on the same street, the distance is -1 ; otherwise, the distance is $w, w>0$.

For the RSU problem of urban road deployment, the RSU deployment vector belongs to $\Omega$. Equation (6) includes two discontinuous objective functions, the set of these two functions is within the range of $R_{k}$, and $f_{1}$ is the sum of all deployed RSUs. In equation (6), after the deployment of RSU, the maximum coverage of the intersection is transformed into the minimization problem. If RSUs are deployed less, then the value of $f_{2}$ is larger and vice versa. If more RSUs are deployed, the value of $f_{2}$ is smaller. 
The two goals are contradictory, namely, the improvement of either goal would cause the decrease of the performance of the other goal, and thus, equation (6) would not reach the optimal value together. It concludes that equation (6) is a two-objective optimization problem, and thus, the problem can be solved by multiobjective evolutionary algorithm.

Traditional multiobjective evolutionary algorithms solve the continuous problem; that is, the values in the initial population are random real ones, and the values in the new population are also real numbers. In this work, RSU deployment $x_{i}$ is 1 when RSU is placed at an intersection and 0 otherwise when RSU is not placed. The individual value in the optimal RSU deployment problem population is 0 or 1 . Then, the algorithm needs to discrete $X$.

$$
\begin{aligned}
& X_{i j}(g)=\left\{\begin{array}{c}
0, S(x)<0.5, \\
1, S(x) \geq 0.5
\end{array}\right. \\
& S(x)=\frac{1}{1+e^{-x}}, X \in R .
\end{aligned}
$$

In equation (8), when $S(x)$ is less than $0.5, X_{i j}(g)$ is equal to 0 (RSU is not deployed at the intersection $j$ ); otherwise, $S(x)$ is greater than or equal to 0.5 , and $X_{i j}(g)$ is equal to 1 (RSU is deployed at intersection $j$ ). In equation (9), the individual $x$ is $X_{i j}(g)$, and $S(x) \in(-1,1)$.

3.2. Fast Nondominated Sorting Approach. Fast nondominant sorting stratifies individuals according to the objective function values of all individuals and sets Pareto levels for each individual. The lower the number of Pareto levels of an individual is, the smaller its fitness value will be, and the greater the probability of inheritance to the next generation will be. All individuals in the same Pareto level are the same as the virtual fitness value. In the same nondominant level, the population diversity of the same dominant level can be improved by judging the crowding degree around the individual.

Assuming the population is $P$, for each individual $p$, we calculate $n_{p}$ and $S_{p}$. In particular, domination count $n_{p}$ is the number of solutions which dominate the solution $p$, and $S_{p}$ is a set of solutions which the solution $p$ dominates, respectively. All individuals in the population are traversed to calculate the NP number and $S_{p}$ set of all individuals.

(1) All population $P$ in the first nondominated front would have their $n_{p}$ as zero, and the individuals with $n_{p}=0$ are placed in the $F_{1}$ set

(2) $P=P-F_{1}$, we continue to find the nondominated solution set of the remaining solutions in $P$; that is, perform $n_{p}=N P-1$ operation on the individually dominated individual $S_{p}$ in $F_{1}$ and place the individual $N P=0$ to $F_{1}$

(3) Repeat the second step until $P$ is empty

Thus, all fronts are $\left\{F_{1}, F_{2} \cdots F_{n}\right\}$.
3.3. Crowding Distance Sorting. The density of solutions can be expressed as average distance of two points on either side of this point along each of the objectives. The crowdingdistance is calculated by sorting the population according to each objective function value in ascending order of magnitude. The crowding distance of the solutions with smallest and largest function values is assigned an infinite distance value. The density of solutions can make more efficient use of high-quality solution sets and ensure the diversity and stability of the population.

Then, crowding distance is defines as

$$
d_{i}=\sum_{k=1}^{m}\left|f_{k}^{i+1}-f_{k}^{i-1}\right| .
$$

When selecting the next generation of individuals for the population, individuals with lower rank are selected, otherwise selecting the solution that is located in larger crowding distance region. In this way, the algorithm can keep approaching PF and keep the diversity of individuals in the population.

After merging the parent population and the newly generated population, the offspring population is selected by elitist guidance strategy through fast nondominated sorting and crowding distance calculation. Since (2) uses the best individual of each generation as the basis vector, it is easy to fall into the local optimal solution, and thus, we tend to select an individual from the top $100 \mathrm{p} \%$ individuals ( $\left.X_{\text {best }}^{p}(g), P \in[5,20]\right)$ in the population as the basic vector $X_{r 1}(g)$ and replace the best individual with individual $X_{\text {best }}^{p}(g)$. The top $40 \%$ of the population is selected as $X_{r 2}(g)$. In order to maintain diversity, an individual $X_{r 3}(g)$ is randomly selected from the population. The improved mutation operation not only accelerates the convergence rate but also increases the diversity of the population, and as shown in equation (11).

$$
\mathbf{V}_{\mathbf{i}}(\mathbf{g})=\mathbf{X}_{\text {best }}^{\mathbf{p}}(\mathbf{g})+F \cdot\left(\mathbf{X}_{\mathrm{r} 2}(\mathbf{g})-X_{r 3}(\mathbf{g})\right)
$$

3.4. MODE-deg Algorithm. Algorithm 1 is the overall MODEdeg framework, Algorithm 2 calculates the sums of the RSU deployment, and Algorithm 3 generates elitist NP individuals, respectively.

Lines 1-2 count the number of RSU deployment. In lines $3-4$, if intersection $i$ deploys RSU, then add $i$ to inter $S$ set. Line 5 calculates total for intersection. In lines 6-10, if intersection $i$ is in the same street as intersection $j$ and is less than or equal to RSU's communication distance, add $j$ to InterS.Finally, in lines 12-14 calculate intersection coverage rate.

In Algorithm 3, the first line executes a fast nondominated sorting to produce the Pareto rank. Line 2 calculates the crowding distance of the population. In line 3, two columns of the Pareto rank and the crowding distance are added to the population. Line 4 sorts by crowding distance in descending order. Line 5 is sorted in an ascending order with the Pareto rank as the keyword. Finally, in line 6, $N P$ elite individuals are selected as the next generation parent population. 


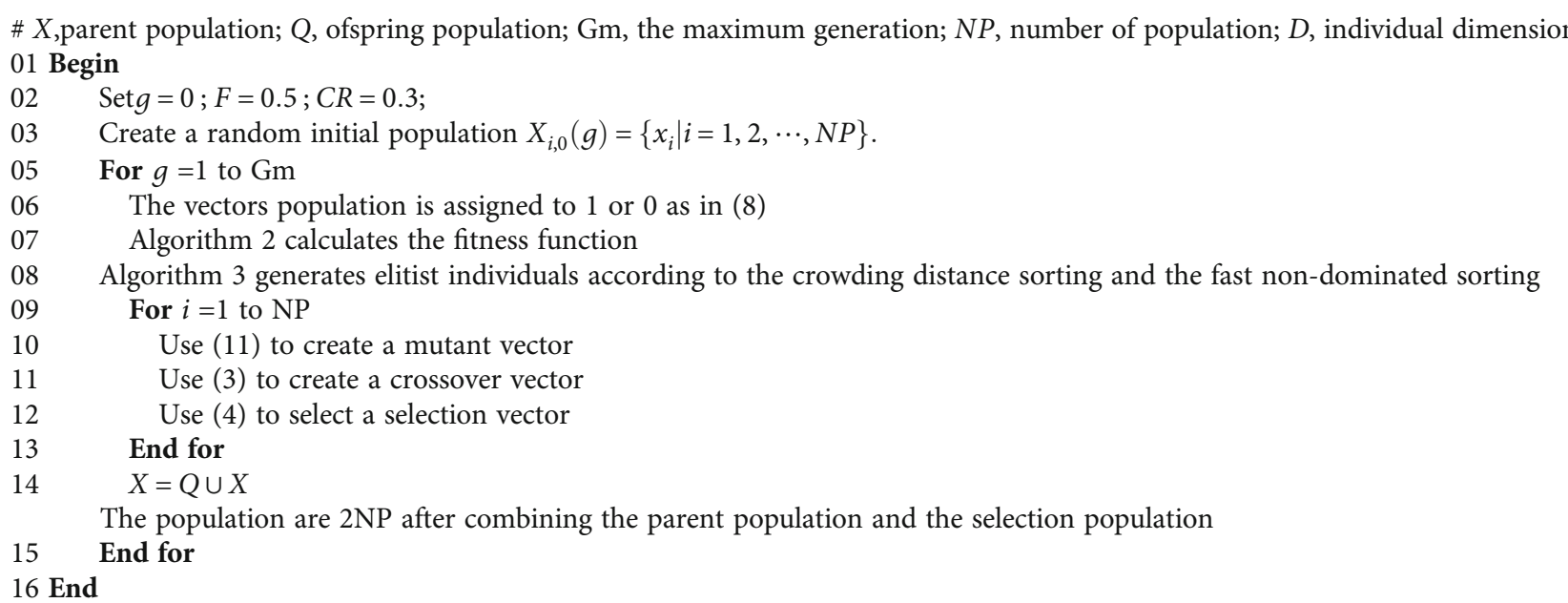

Algorithm 1: MODE-deg outline.

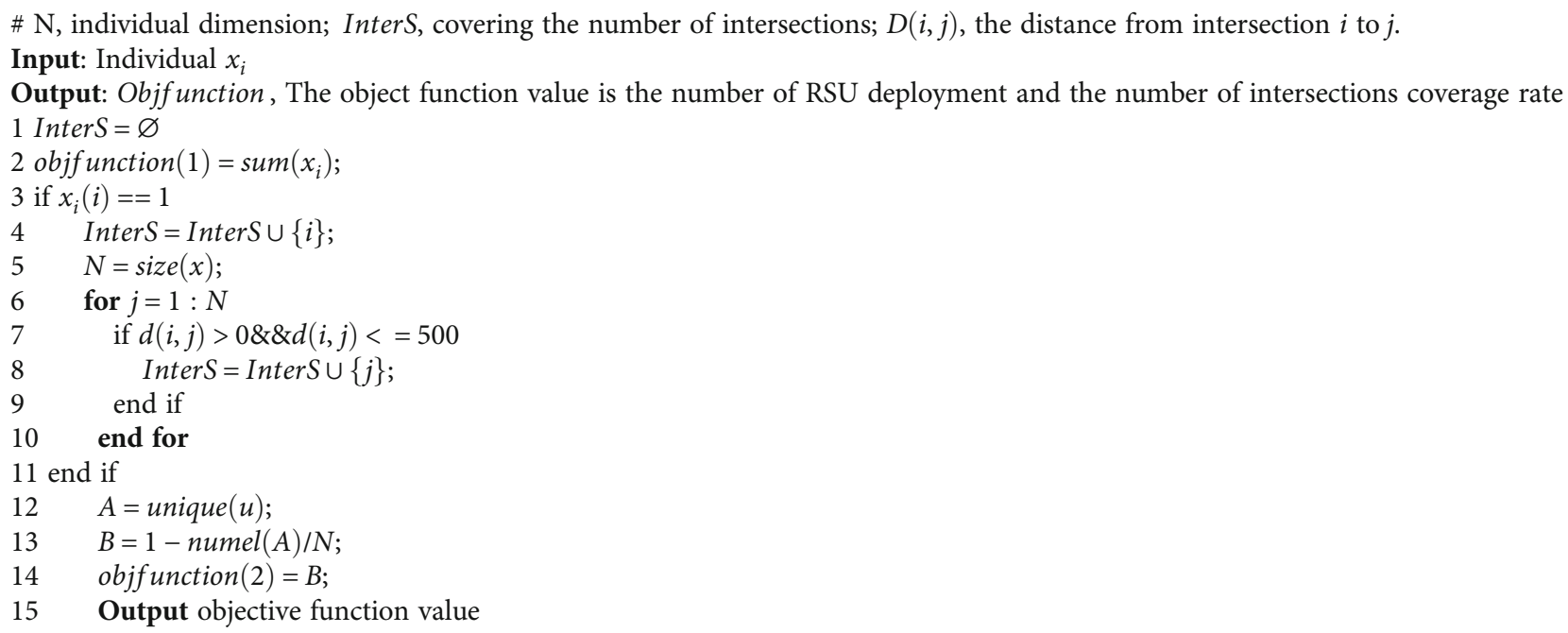

Algorithm 2: Calculating the RSU deployment objective function value.

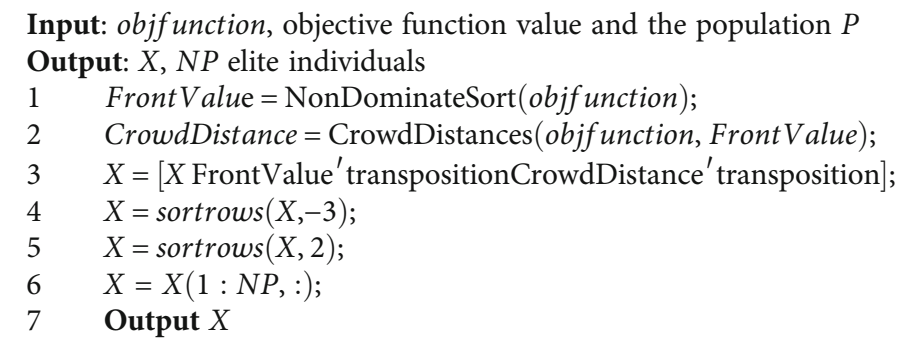

Algorithm 3: Generating the elitist NP individuals. 


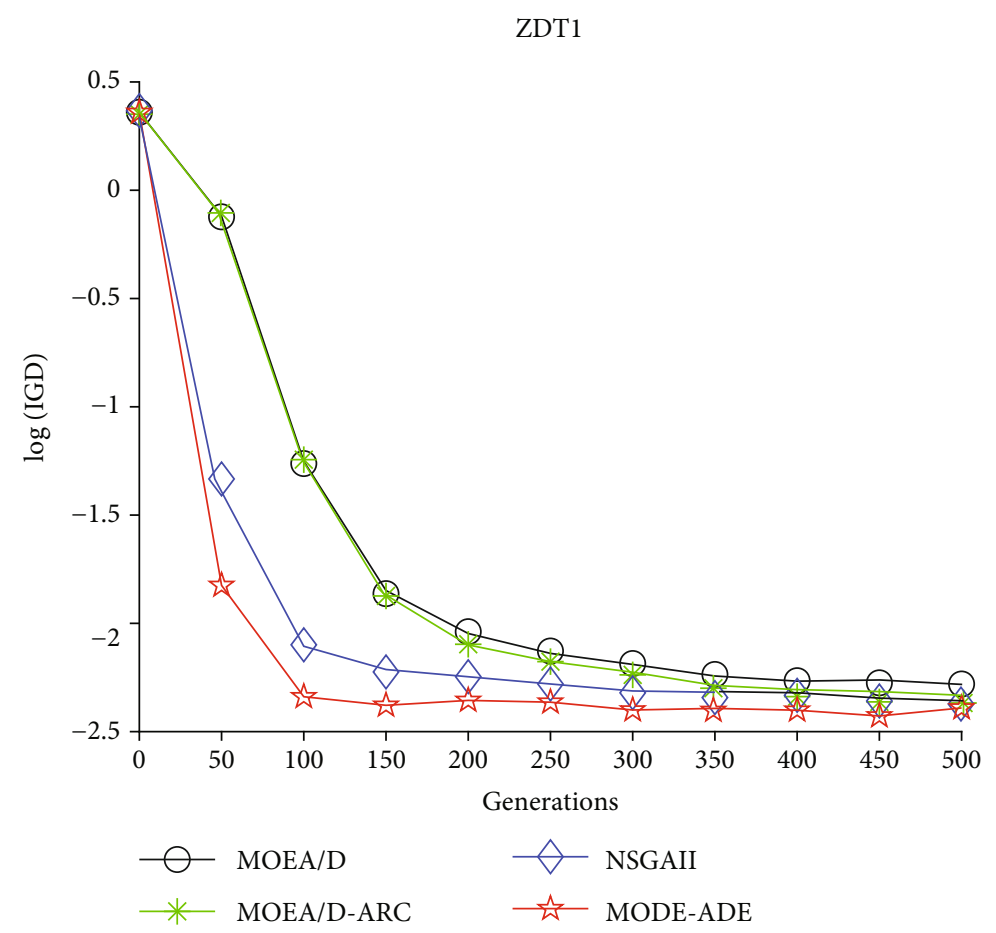

(a)

ZDT2

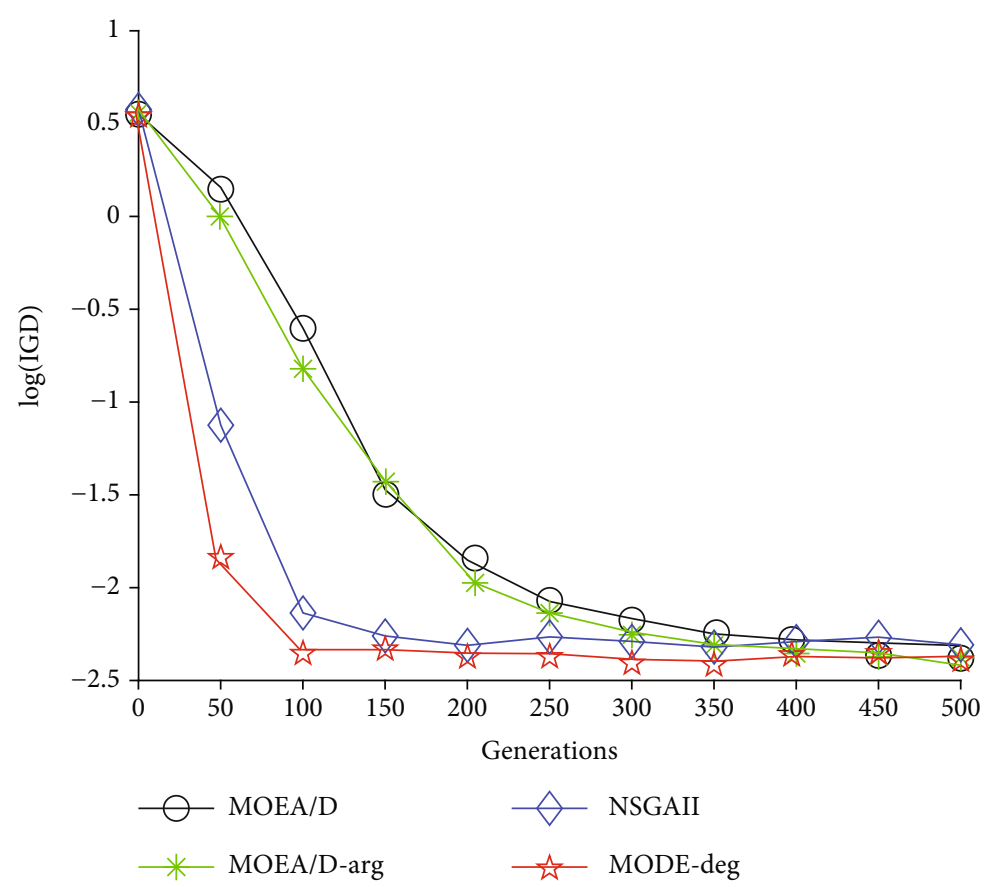

(b)

Figure 2: Continued. 


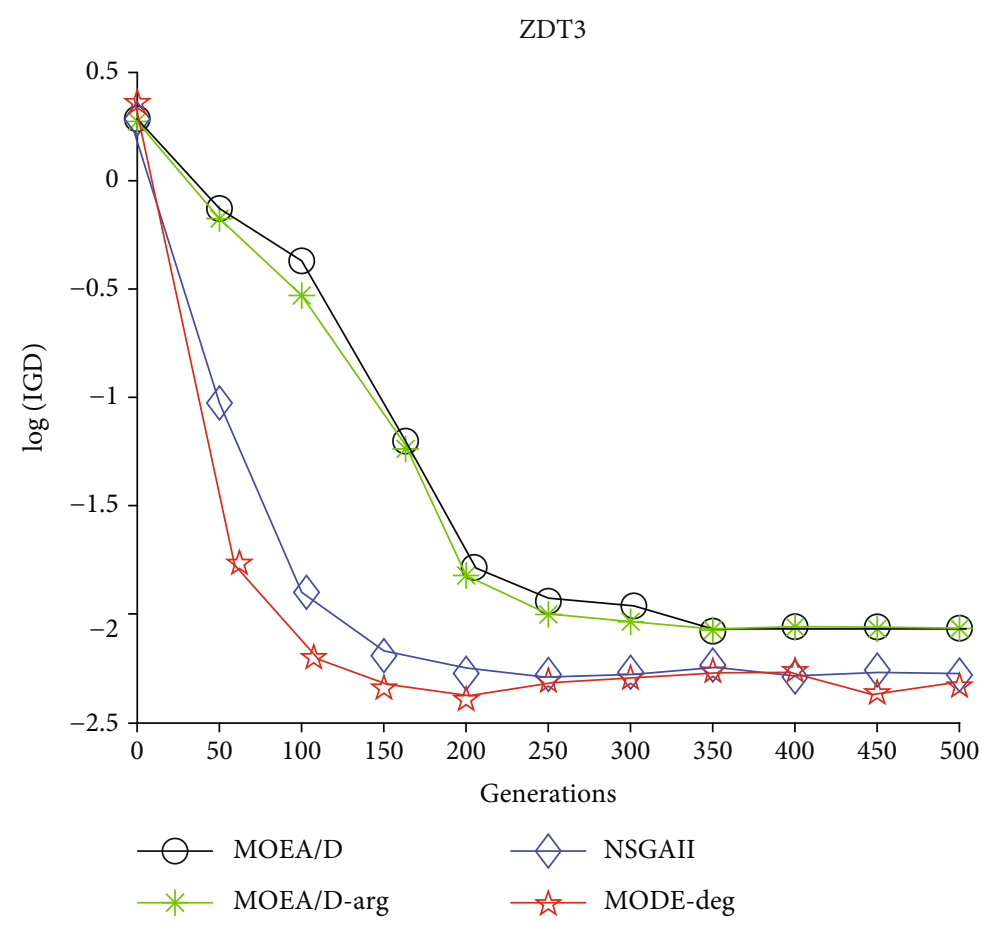

(c)

ZDT4

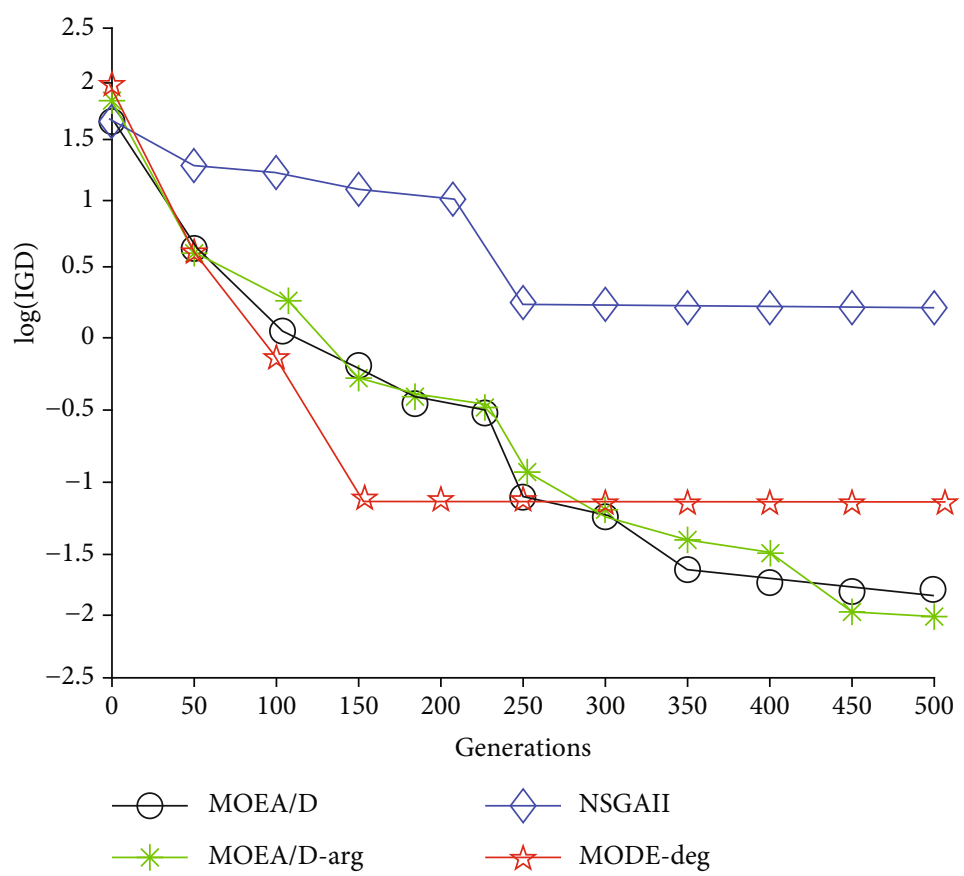

(d)

Figure 2: Continued. 


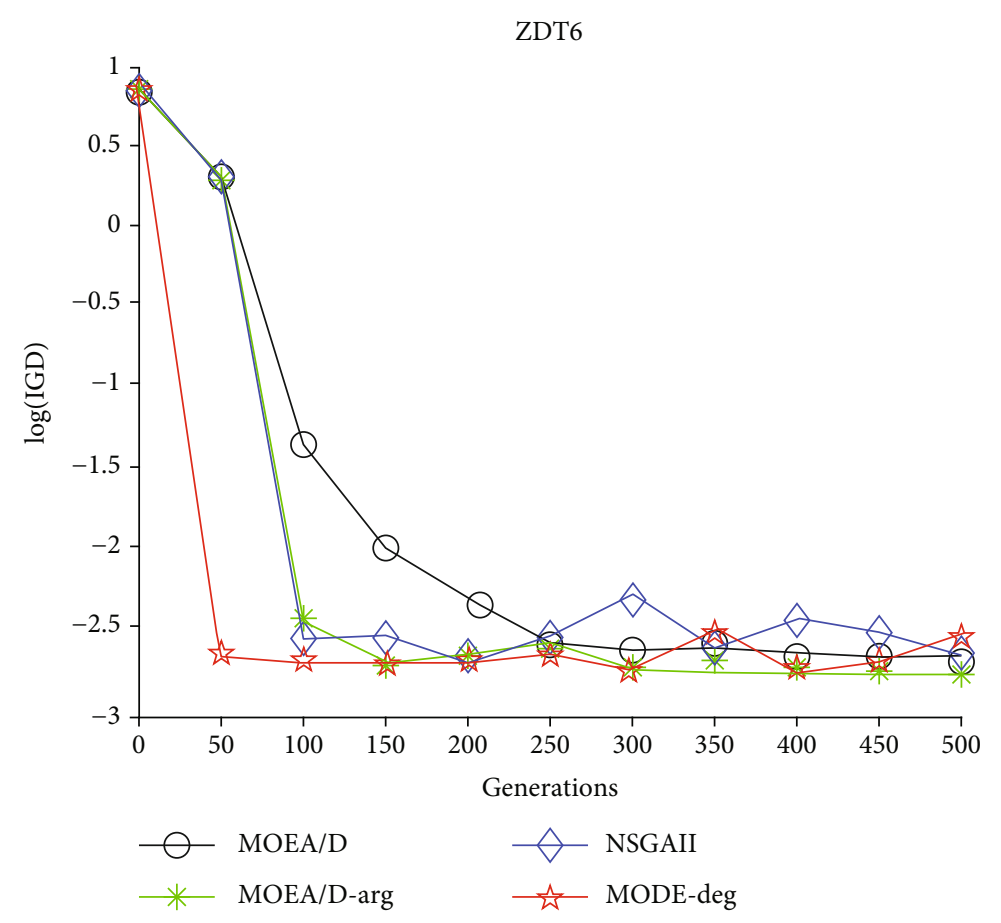

(e)

Figure 2: Mean IGD convergence comparison.

TABLE 1: Comparison between MODE-deg and other MOEAs based on IGD.

\begin{tabular}{|c|c|c|c|c|c|}
\hline Problem & IGD & MOEA/D-arg & MOEA/D & NSGA-II & MODE-deg \\
\hline \multirow{3}{*}{ ZDT1 } & Mean & $7.54 E-03$ & $9.16 E-03$ & $5.30 E-03$ & $4.36 E-03$ \\
\hline & Std & $8.33 E-04$ & $1.09 E-03$ & $4.18 E-04$ & $2.28 E-04$ \\
\hline & Rank & 3 & 4 & 2 & 1 \\
\hline \multirow{3}{*}{ ZDT2 } & Mean & $6.22 E-03$ & $9.03 E-03$ & $5.10 E-03$ & $4.54 E-03$ \\
\hline & Std & $4.20 E-03$ & $1.11 E-02$ & $3.26 E-04$ & $2.63 E-04$ \\
\hline & Rank & 3 & 4 & 2 & 1 \\
\hline \multirow{3}{*}{ ZDT3 } & Mean & $9.44 E-03$ & $5.76 E-02$ & $5.63 E-03$ & $4.82 E-03$ \\
\hline & Std & $7.20 E-04$ & $6.57 E-02$ & $3.00 E-04$ & $2.81 E-04$ \\
\hline & Rank & 3 & 4 & 2 & 1 \\
\hline \multirow{3}{*}{ ZDT4 } & Mean & $2.15 E-01$ & $3.49 E-01$ & 4.83 & $3.45 E-02$ \\
\hline & Std & $1.13 E-01$ & $1.46 E-01$ & 3.38 & $5.36 E-02$ \\
\hline & Rank & 2 & 3 & 4 & 1 \\
\hline \multirow{3}{*}{ ZDT6 } & Mean & $2.33 E-03$ & $2.20 E-03$ & $3.30 E-03$ & $2.96 E-03$ \\
\hline & Std & $3.33 E-04$ & $1.93 E-04$ & $6.53 E-04$ & $3.77 E-04$ \\
\hline & Rank & 2 & 1 & 4 & 3 \\
\hline Total final & & 13 & 16 & 14 & 7 \\
\hline Rank & & 2 & 4 & 3 & 1 \\
\hline
\end{tabular}

\section{Simulation Results and Discussions}

In this section, MODE-deg is applied to minimize a set of 5 scalable benchmark functions of dimensions $D=30$ or 10. First, we state five test functions and inverted genera- tional distance (IGD) indicators to measure the performance of the MOEA algorithm. Next, we compare MODE-deg with three elitist MOEAs: NSGA-II, MOEA/D, and MOEA/D-arg. Finally, the validity of MOEA/D-deg is verified. 


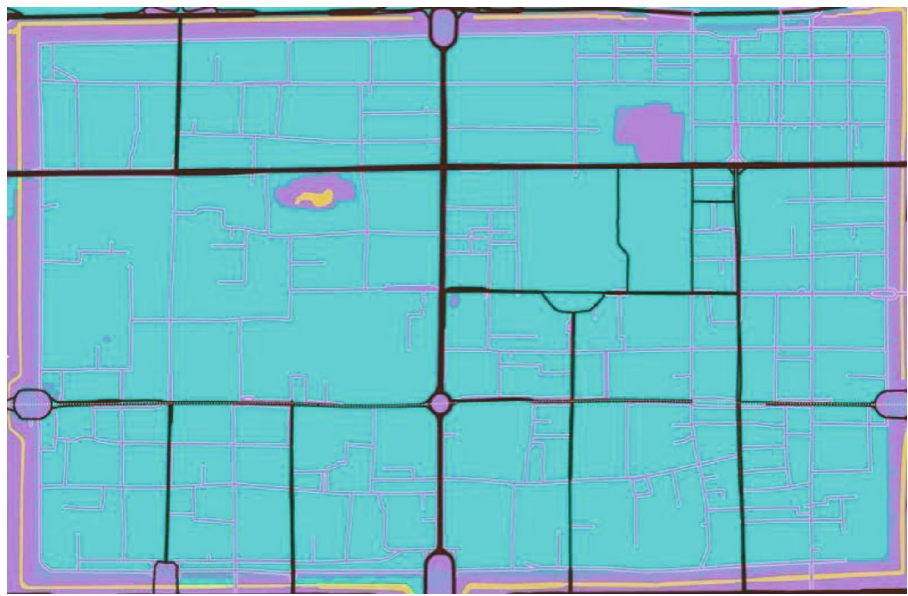

Figure 3: Urban traffic map of Xi'an.

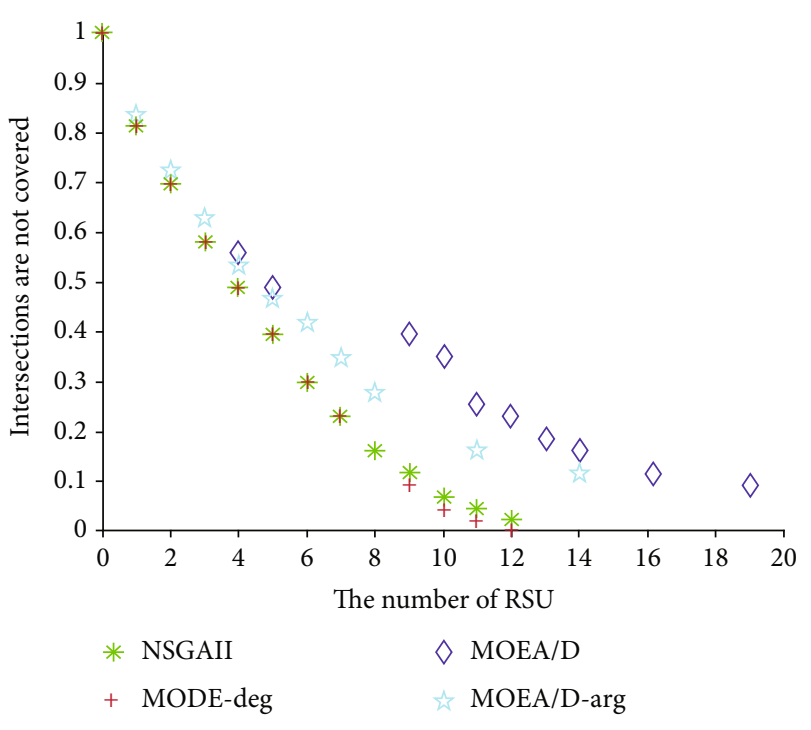

FIGURE 4: Nondominant solution set of RSU deployment.

4.1. Performance Metric. The IGD is used to assess both convergence and diversity, and formula is expressed as:

$$
\operatorname{IGD}(P, Q)=\frac{\sum_{v \in P} d(v, Q)}{|P|},
$$

where $|P|$ is a set of points evenly distributed on the true Pareto front, $|P|$ is the number of distribution points on the $\mathrm{PF},|Q|$ is the optimal Pareto optimal solution set obtained by the algorithm, and $d(v, Q)$ is the minimum Euclidean distance between individual $|v|$ in $P$ and population $|Q|$, respectively. The smaller the value is, the better the algorithm's convergence is. However, when the diversity of the algorithm is poor and most individuals in the population are concentrated in a narrow area, it can be seen from the equation that $d(v, Q)$ of many individuals will be large, so the diversity performance of the algorithm can be evaluated. Therefore, IGD evaluates the comprehensive performance of the algorithm by calculating the average value of the minimum distance between the point set on the real Pareto frontier and the acquired population in the target space point set.

4.2. Hypervolume. Hypervolume (HV) is the volume of the region in the target space enclosed by the PS obtained by the algorithm and the reference points. The higher the HV value is, the better the comprehensive performance of the algorithm is. $\delta$ is the Lebesgue measure used to measure volume, $|s|$ is the number of a dominant solution set, and $V_{i}$ is the $\mathrm{HV}$ of the reference point and the $i$ th solution in the solution set, respectively. The formula is expressed as:

$$
\mathrm{HV}=\delta\left(\bigcup_{i=1}^{|s|} v_{i}\right)
$$

$\mathrm{HV}$ has the following advantages: convergence and diversity are evaluated simultaneously, and no PF or reference set is required. Nevertheless, benefits never come without the cost, e.g., high computational complexity. Meanwhile, the selection of reference points determines the accuracy of the $\mathrm{HV}$ evaluation metrics.

4.3. Results and Discussion. We firstly compare the mean IGD-metric values of MODE-arg algorithm with other three MOEAs in five test functions of ZDT. Taking the urban traffic map of Xi'an as the research object, we apply four MOEA algorithms to test MODE-deg on ZDT problems and the RSU deployment problems, respectively. For simplicity, we only study two objective optimization problems.

The experiments are carried out in a desktop PC with Intel Core (TM) i7-4970 $4 \mathrm{CPU} 3.60 \mathrm{GHz}$ and $16 \mathrm{~GB}$ RAM. In the parameter setting, the population size of $N P$ is 100 for the ZDT problems and 40 for the RSU deployment problems, respectively. Furthermore, individual dimension is 30 or 10 for the ZDT, individual dimension is 43 , the max generation is 500 , and each problem is set as 20 . In the control parameters, $C R$ is $0.3, F$ is $0.5, T$ is 20 in MOEA/D, and $T_{r}$ (replacing the number in the neighbourhood in MOEA/D-arg) is exponential growth, respectively. 
TABLE 2: RSU deployment nondominant solution set and coverage rate.

\begin{tabular}{|c|c|c|c|c|c|c|c|}
\hline \multicolumn{2}{|c|}{ MODE-deg } & \multicolumn{2}{|c|}{ NSGA-II } & \multicolumn{2}{|c|}{ MOEA/D } & \multicolumn{2}{|c|}{ MOEA/D-arg } \\
\hline RSU & Coverage & RSU & Coverage & RSU & Coverage & RSU & Coverage \\
\hline$\overline{1}$ & $18.6 \%$ & 1 & $8.6 \%$ & 1 & - & - & - \\
\hline 2 & $30.2 \%$ & 2 & $0.2 \%$ & 2 & $7.9 \%$ & - & - \\
\hline 3 & $41.8 \%$ & 3 & $41.8 \%$ & 3 & $37.2 \%$ & - & - \\
\hline 4 & $51.1 \%$ & 4 & $51.1 \%$ & 4 & $46.5 \%$ & - & - \\
\hline 5 & $60.4 \%$ & 5 & $60.4 \%$ & 5 & $51.2 \%$ & - & - \\
\hline 6 & $69.7 \%$ & 6 & $69.7 \%$ & 6 & $58.1 \%$ & - & - \\
\hline 7 & $76.7 \%$ & 7 & $76.7 \%$ & 7 & $62.8 \%$ & 7 & $53.5 \%$ \\
\hline 8 & $83.7 \%$ & 8 & $83.7 \%$ & 8 & $65.1 \%$ & 8 & $62.8 \%$ \\
\hline 9 & $90.6 \%$ & 9 & $88 \%$ & 9 & $69.8 \%$ & 11 & $81.4 \%$ \\
\hline 10 & $95.3 \%$ & 10 & $93 \%$ & - & - & 13 & $86.0 \%$ \\
\hline 11 & $97.6 \%$ & 11 & $96.3 \%$ & - & - & - & - \\
\hline 12 & $100 \%$ & 12 & $97.8 \%$ & - & - & - & - \\
\hline 13 & - & 13 & $100 \%$ & 15 & $90.7 \%$ & 17 & $90.7 \%$ \\
\hline
\end{tabular}

TABle 3: Running time and HV comparison between MODEs.

\begin{tabular}{lcccc}
\hline & MOEA/D-arg & MOEA/D & NSGA-II & MODE-deg \\
\hline HV & 0.48148 & 0.34821 & 0.59597 & 0.61322 \\
The elapsed time (s) & 28.3395 & 1.4574 & 1.5364 & 1.7696 \\
\hline
\end{tabular}

Figure 2 is the IGD convergence comparison between MODE-deg, MOEA/D, MOEA/D-arg, and NSGAII. As can be seen from Figure 2, in Figures 2(a)-2(c), MODE-deg has the fastest convergence rate on ZDT1, ZDT2, and ZDT3 test functions, and the optimal solution set is also superior to MOEAs. In Figure 2(e), MODE-deg has the fastest convergence rate in the early stage, and PS is close to other MOEAs on ZDT6. In Figure 2(d), MODE-deg has a fast convergence rate in the early stage, and $\mathrm{PF}$ is inferior to MOEA/D and MOEA/D-arg on ZDT4.

The mean IGD-metric values of MODE-deg in 20 independent runs on ZDT are given in Table 1 . Table 1 shows that MODE-deg ranks first among the four algorithms, which indicates that MODE-deg can generate good PF with good convergence and diversity on the five test functions.

4.4. Applications to Solve RSU. We take the urban traffic map of Xi'an as the research object and as shown in Figure 3. The lines in the map are urban roads with 43 main intersections. And we place RSUs at the intersections and use the above four algorithms, respectively, to solve the nondominated solution set.

In Figure 4, the abscissa axis is the number of deployed RSUs, and the ordinate is 1- (RSU coverage rate). The nondominant solution set of MODE-deg and NSGAII is superior to that of MOEA/D and MOEA/D-arg, and the coverage of MODE-deg and NSGAII is the same when RSUs are less than 9. When MODE-deg and NSGAII deploy 9-12 RSUs, they have different coverage rates. In particular, when MODE-deg deploys 12 RSUs, the algorithm achieves $100 \%$ coverage. The nondominant solution set of the four algorithms is shown in the following table.

As shown in Table 2, MOEA/D and MOEA/D-arg can only obtain the number and coverage of partial RSUs. When MOEA/D-arg is deployed with 11 RSUs, coverage of more than $80 \%$ can be achieved; when MOEA/D-arg is deployed with 17 RSUs, coverage of more than $90 \%$ can be achieved but not 100\%. MOEA/D deployments with 15 RSUs can achieve more than $90 \%$ coverage, while algorithms cannot achieve $100 \%$ coverage. MODE-deg and NSGA-II deploy eight RSUs to achieve $80 \%$ coverage. In particular, MODE-deg can achieve $100 \%$ coverage with 10 RSUs, while NSGA-II can achieve more than $90 \%$ coverage with 10 RSUs.

In Table 3, the program is run 30 times for each algorithm, and the population has evolved 300 generations. Among the four algorithms, the HV value of MODE-deg is the largest, which proves that the optimality of nondominant solution set obtained by this algorithm is better.

More especially, the time complexity of MODE-deg algorithm and NSGA-II is $O\left(m n^{2}\right)$, where the number of objective function is $m$ and $n$ is the population size. However, when selecting $\mathbf{X}_{\text {best }}^{\mathbf{p}}(g)$ individuals in the MODE-deg algorithm, the population is sorted from high to low in the descending order of crowding distance and the ascending order of Pareto ranks (Algorithm 2), and thus, the running time of MODE-deg is longer than NSGA-II. The time complexity of MOEA/D is $O(m n t)$, where the neighbourhood size is $t$ and the running time is the shortest. It follows that MOEA/D-arg is an improvement of MOEA/D algorithm, which changes the neighbourhood substitution of offspring 


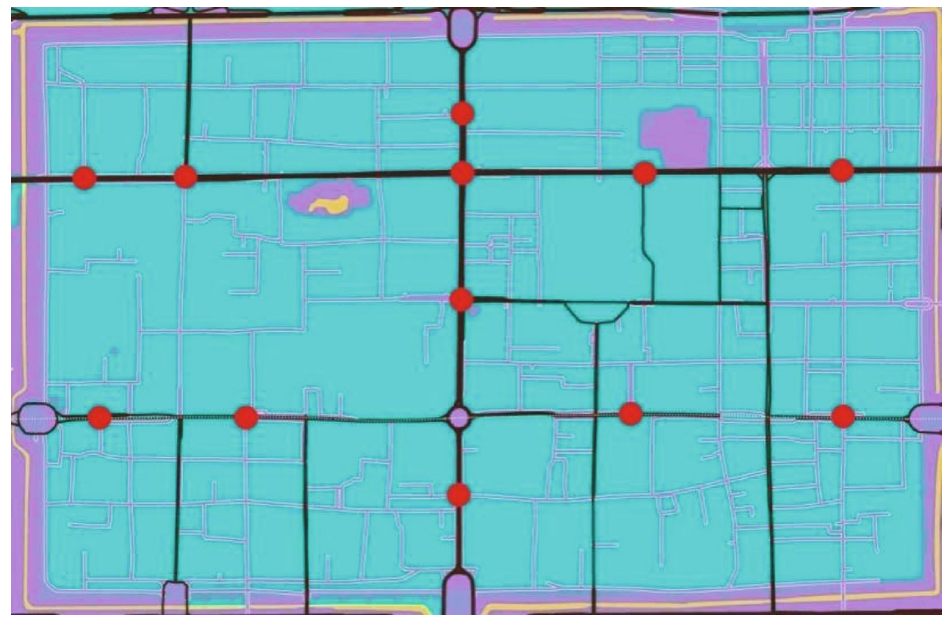

FIgURE 5: RSU deployment.

individuals into global substitution, improving the individual utilization rate but sacrificing the running time. Moreover, the time complexity of MOEA/D-arg is $O\left(m n^{2} t\right)$, and its running time is the largest.

In Figure 5, the red marks are the RSU locations in the map, and a total of 12 RSUs are deployed to achieve $100 \%$ intersection coverage of main roads.

\section{Conclusion}

This paper studies the problem of RSU deployment in cities and proposes a new algorithm named MODE-deg. After the individual is normalized to 0 or 1 , nondominated ordering is performed on individuals, and crowding distance is calculated, and excellent individuals are selected for guidance to accelerate the convergence speed. The performance of MODE-deg algorithm is evaluated by comparing with other three multiobjective evolutionary algorithms on five test problems. Experimental results show that MODE-deg algorithm produces good convergence and diverse Pareto set, which can accelerate the convergence speed. Finally, MODE-deg and the other three MOEDs are applied to the established RSU model, showing that MODE-deg can achieve greater coverage with fewer RSUs. In the future, we will study how to improve the communication success rate of mobile vehicles in the coverage area.

\section{Data Availability}

The data used to support the findings of this study are included within the article.

\section{Conflicts of Interest}

The authors declare no conflicts of interest.

\section{Acknowledgments}

This work was supported in part by the National Natural Science Foundation of China under Grants 61801379 and 61803301.

\section{References}

[1] M. Jain and R. Saxena, "Overview of VANET: requirements and its routing protocols," in Proceedings of IEEE international Conference on Communication \& Signal Processing (ICCSP), pp. 1957-1961, Chennai, India, 2017.

[2] Q. Yang, L. Wang, W. Xia, Y. Wu, and L. Shen, "Development of on-board unit in vehicular ad-hoc network for highways," in 2014 International Conference on Connected Vehicles and Expo (ICCVE), pp. 457-462, Vienna, Austria, 2014.

[3] S. I. Sou and O. K. Tonguz, "Enhancing VANET connectivity through roadside units on highways," IEEE Transactions on Vehicular Technology, vol. 60, no. 8, pp. 3586-3602, 2011.

[4] Y. Li, L. Zhu, H. Wang, F. R. Yu, and S. Liu, "A cross-layer defense scheme for edge intelligence-enabled CBTC systems against MitM attacks," IEEE Transactions on Intelligent Transportation Systems, vol. 22, no. 4, pp. 2286-2298, 2021.

[5] L. Zhu, Y. Li, F. R. Yu, B. Ning, T. Tang, and X. Wang, "Crosslayer defense methods for jamming-resistant CBTC systems," EEE Transactions on Intelligent Transportation Systems, vol. 22, no. 22, pp. 7266-7278, 2021.

[6] R. Xie, Q. Tang, S. Qiao, H. Zhu, F. Richard Yu, and T. Huang, "When serverless computing meets edge computing: architecture, challenges, and open issues," IEEE Wireless Communications, pp. 1-8, 2021.

[7] Z. Wang, J. Zheng, Y. Wu, and N. Mitton, "A centrality-based RSU deployment approach for vehicular ad hoc networks," in 2017 IEEE International Conference on Communications (ICC), pp. 1-5, Paris, France, 2017.

[8] R. Cai, Y. Feng, D. He, Y. Xu, Y. Zhang, and W. Xie, "A combined cable-connected RSU and UAV-assisted RSU deployment strategy in V2I communication," in ICC 2020 - 2020 IEEE International Conference on Communications (ICC), pp. 1-6, Ireland, Dublin, 2020.

[9] D. Kim, Y. Velasco, W. Wang, R. N. Uma, R. Hussain, and S. Lee, "A new comprehensive RSU installation strategy for cost-efficient VANET deployment," IEEE Transactions on Vehicular Technology, vol. 66, no. 5, pp. 4200-4211, 2017.

[10] M. Al Shareeda, A. Khalil, and W. Fahs, "Towards the optimization of road side unit placement using genetic algorithm," in In Proceedings of IEEE International Arab Conference on Information Technology (ACIT), pp. 1-5, Werdanye, Lebanon, 2018. 
[11] Z. W. Lamb and D. P. Agrawal, "Data-driven approach for targeted RSU deployment in an urban environment," in 2017 IEEE Intelligent Vehicles Symposium (IV), pp. 1916-1921, Los Angeles, CA, USA, 2017.

[12] S. Jian and Y. Yang, "RSU location optimization model under Vii environment," International Journal on Information, vol. 26, no. 4, pp. 394-402, 2011.

[13] Y. Ni, J. He, L. Cai, J. Pan, and Y. Bo, "Joint roadside unit deployment and service task assignment for Internet of Vehicles (IoV)," IEEE Internet of Things Journal, vol. 6, no. 2, pp. 3271-3283, 2018.

[14] J. Chi, Y. Jo, H. Park, and S. Park, "Intersection-priority based optimal RSU allocation for VANET," in 2013 Fifth International Conference on Ubiquitous and Future Networks (ICUFN), pp. 350-355, Da Nang, Vietnam, 2013.

[15] X. Cao, Q. Cui, S. Zhang, X. Jiang, and N. Wang, "Optimization deployment of roadside units with mobile vehicle data analytics," in 2018 24th Asia-Pacific Conference on Communications (APCC), pp. 358-363, Ningbo, China, 2018.

[16] X. Cao, Q. Cui, S. Zhang, X. Jiang, and N. Wang, "Optimal and greedy algorithms for the one-dimensional RSU deployment problem with new model," IEEE Transactions on Vehicular Technology, vol. 67, no. 8, pp. 7643-7657, 2018.

[17] O. Trullols, M. Fiore, C. Casetti, C. F. Chiasserini, and J. M. Barcelo Ordinas, "Planning roadside infrastructure for information dissemination in intelligent transportation systems," Computer Communications, vol. 33, no. 4, pp. 432-442, 2010.

[18] C. Lochert, B. Scheuermann, C. Wewetzer, A. Luebke, and M. Mauve, "Data aggregation and roadside unit placement for a VANET traffic information system," in Proceedings of ACM International Workshop on Vehicular Inter-Networking, Association for Computing Machinery, pp. 58-65, New York, NY, USA, 2008.

[19] D. Ou, Y. Yang, L. Xue, and D. Dong, “Optimal connectivitybased deployment of roadside units for vehicular networks in urban areas," Transportation Research Record Journal of the Transportation Research Board, vol. 2559, no. 1, pp. 46-56, 2016.

[20] H. R. Eftekhari, A. J. Bashirzadeh, and M. Ghatee, "Binary programming model to optimize RSU placement for information dissemination," in 2015 International Conference on Connected Vehicles and Expo (ICCVE), pp. 112-115, Shenzhen, China, 2015.

[21] S. Anbalagan, A. K. Bashir, G. Raja et al., "Machine learningbased efficient and secure RSU placement mechanism for software defined-IoV," IEEE Internet of Things Journal, vol. 8, no. 18, pp. 13950-13957, 2021.

[22] M. Sankaranarayanan, C. Mala, and S. Mathew, "Genetic algorithm based efficient RSU distribution to estimate travel time for vehicular users," in 2015 Second International Conference on Soft Computing and Machine Intelligence (ISCMI), pp. 30-34, Hong Kong, China, 2015.

[23] L. Zhu, H. Liang, H. Wang, B. Ning, and T. Tang, "Joint security and train control design in blockchain empowered CBTC system," IEEE Internet of Things Journal, 2021.

[24] E. Zitzler and L. Thiele, "Multiobjective optimization using evolutionary algorithms-a comparative case study," in Proceedings of International Conference on Parallel Problem Solving from Nature, pp. 292-301, Berlin, Germany, 1998.
[25] R. Storn and K. Price, "Differential evolution a simple and efficient heuristic for global optimization over continuous apaces," Journal of Global Optimization, vol. 11, no. 4, pp. 341-359, 1997.

[26] N. Srinivas and K. Deb, "Multiobjective function optimization using nondominated sorting genetic algorithms," IEEE Transactions on Evolutionary Computation, vol. 2, no. 3, pp. 221248, 1994.

[27] K. Deb, A. Pratap, S. Agarwal, and T. Meyarivan, "A fast and elitist multiobjective genetic algorithm: NSGA-II," IEEE Transactions on Evolutionary Computation, vol. 6, no. 2, pp. 182-197, 2002.

[28] J. Horn, N. Nafpliotis, and D. E. Goldberg, "A nicked Pareto genetic algorithm for multi-objective optimization," in Proceedings of the First IEEE Conference on Evolutionary Computation. IEEE World Congress on Computational Intelligence, pp. 82-87, Orlando, FL, USA, 1994.

[29] E. Zitzler, M. Laumanns, and L. Thiele, "SPEA2: improving the strength Pareto evolutionary algorithm," Technical Report Gloriastrasse, vol. 103, pp. 283-290, 2001.

[30] Qingfu Zhang and Hui Li, "MOEA/D: a multiobjective evolutionary algorithm based on decomposition," IEEE Transactions on Evolutionary Computation, vol. 11, no. 6, pp. 712-731, 2007.

[31] Z. Wang, Q. Zhang, A. Zhou, M. Gong, and L. Jiao, “Adaptive replacement strategies for MOEA/D," IEEE Transactions on Cybernetics, vol. 46, no. 2, pp. 474-486, 2016.

[32] H. Liu, F. Gu, and Q. Zhang, "Decomposition of a multiobjective optimization problem into a number of simple multiobjective subproblems," IEEE Transactions on Evolutionary Computation, vol. 18, no. 3, pp. 450-455, 2014.

[33] N. Beume, B. Naujoks, and M. Emmerich, "SMS-EMOA: multiobjective selection based on dominated hypervolume," European Journal of Operational Research, vol. 181, no. 3, pp. 1653-1669, 2007.

[34] E. Zitzler and S. Künzli, "Indicator-based selection in multiobjective search," in Proceedings of International Conference on Parallel Problem Solving from Nature, pp. 832-842, Berlin, Germany, 2004. 